\title{
Reviewers for Kew Bulletin Volume 68
}

We are grateful to the following people who reviewed articles for Kew Bulletin during the years 2012 - 2013.
D. Albach
F. Almeda
M. Nee
D. Austin
M. Barkworth
H. Noltie
S. O'Kane
J. Batista
H. A. Pederson
C. Bayer
C. Pendry
P. de Block
K. Redden
P. Brandham
S. Renvoize
M. Callmander
E. Roalson
R. Chung
Y. F. Deng
M. Dillon
E. Forero
G. Romao
D. Salariato
M. Santos
S. Freire
A. Sartori
M. Gottschling
R. M. K. Saunders
T. Henkel
B. Jackes
P. Jørgensen
R. Kiew
N. Snow
S. Suddee
P. Suksathan
L. J. G. van der Maesen
P. Van Welzen
J. Kirkbride
A. Vizzini
H. Kurzweil
R. Vogt
I. Larridon
S.J. Li
S. Lindsay
J. Wiersema
K. Wilson
A. M. Muasya
J. R. I. Wood
C. Zickel 